\title{
A TRAINING NETWORK FOR GREEN DATA CENTRES OPERATIONS MANAGEMENT THROUGH STAFF EXCHANGE
}

\author{
H. Lee', C. Pitelis', K. Kim², Y. Choi², B. Dinev ${ }^{3}$, Y. Yonkov³, H. Hocaoglu4, T. \\ Gozel $^{4}$, S. Yenice ${ }^{5}$, M. Aktas ${ }^{5}$ \\ ${ }^{1}$ Brunel University London (UK) \\ ${ }^{2}$ LK Knowledge Engineering LTD (UK) \\ ${ }^{3}$ David Holding (Bulgaria) \\ ${ }^{4}$ Gebze Technical University (Turkey) \\ ${ }^{3}$ Turksat (Turkey)
}

\begin{abstract}
This paper introduces the GREENDC project which was funded by EU through H2020 Marie Skłodowska-Curie actions and aims at training early stage researchers for green data centre operations management. The project also enable experienced academic researchers to apply their research outcomes to real world data centre operations management processes. The project also contribute to greener data centres by developing a decision support tool that help data centre managers predict energy demands better and evaluate strategies to minimize energy waste and minimize CO2 emissions. GREENDC adopts non-linear energy forecasting model and provide a simulation tool based on dynamic simulation model to allow data centre managers conduct what-if analysis considering factors for energy demands and supply. GREENDC is implemented through knowledge exchange between two academic partners and two industrial partners. Academic partners transfer knowledge on non-linear energy demand forecasting and dynamic simulation to industrial partners while industrial partners transfer their knowledge on data centre operations through secondment activities. The outcome of the GREENDC activities are expected to reduce CO2 emissions and energy waste due to non-optimised energy load balancing from large number of data centres across the Europe. This paper explains how the knowledge exchange scheme make impacts on the career development of early stage researchers from academia and industry.
\end{abstract}

Keywords: training network, green data centre, operations management, knowledge exchange, decision support systems.

\section{INTRODUCTION}

Data centres (DCs) are one of the major energy consumers and source of $\mathrm{CO} 2$ emissions globally. This paper introduces the GREENDC project that addresses the growing challenge by developing and exploiting a novel approach to forecasting energy demands. The project will bring together five leading academic and industrial partners with the overall aim of reducing energy consumption and $\mathrm{CO} 2$ emissions in specific national DCs. It will implement a total of 163 person-months staff and knowledge exchanges between industry and academic partners. More specifically, knowledge of data centres operations will be transferred from industry to academic partners, whereas simulation based optimization for best practice of energy demand control will be transmitted from academia to industry through the knowledge transfer scheme.

In recent years, the use of the information and communications technologies (ICT), comprising communication devices and/or applications, DCs, internet infrastructure, mobile devices, computer and network hardware and software and so on, has increased rapidly. Internet service providers such as Amazon, Google, Yahoo, etc., representing the largest stakeholders in the IT sector, constructed a large number of geographically distributed internet data centers (IDCs) to satisfy the growing demand and providing reliable low latency services to customers[1],[2]. These IDCs have a large number of servers, large-scale storage units, networking equipment, infrastructure, etc., to distribute power and provide cooling. The number of IDCs owned by the leading IT companies is drastically increasing every year. As a result, the number of servers needed has reached an astonishing number. According to recent reports [3], the approximate number of servers large companies possess are as follows: Google ( $\approx 1$ million), Microsoft (>1 million), Akamai $(\approx 127 \mathrm{~K})$, INTEL $(\approx 100 \mathrm{~K})$, facebook $(>300 \mathrm{~K})$, ebay( $\approx 60 \mathrm{~K})$, Rackspace $(\approx 100 \mathrm{~K})$ [2]. With rapid increase in demand for internet service providers, the energy used by IDCs has been skyrocketing due to the large number of hosted servers and 
associated workload[4],[5]. The global energy cost for enterprise and DCs power and cooling reached $\$ 30$ billion in year 2008 only [6]. According the EPA report published in 2007, IDCs consumed about 61 billion kilowatt-hour $(\mathrm{kWh})$ in 2006 for a total electricity cost of about $\$ 4.5$ billion [7]. This means that IDCs consumed $1.5 \%$ of total U.S. electricity consumption. It was projected that this would rise to $3 \%$ in 2011 or in other words about 120 billion $\mathrm{kWh}$ equivalent to the average consumption of a city with 11.6 million households. In reports published in 2013 [8] it is shown how the IT sector is consuming about $10 \%$ of the world's electricity generation. According to JRC [9], IDCs consume already about $50 \mathrm{TWh}$, which is projected to increase to $100 \mathrm{TWh}$ by 2020 . DCs electricity consumption in Western Europe alone was 56 TWh in 2007 and is projected to rise to $104 \mathrm{TWh}$ in 2020 [9].

Due to large amount of energy implied and the related cost, IDCs can make a significant contribution to the energy efficiency by reducing energy consumption and power management of IT ecosystems. This is why most researchers focus on reducing power consumption of IDCs [2],[8],[13],[14]. One of the ways to reduce power consumption of IDCs is to optimise load-balancing among servers to prevent unnecessary overloads to part of the servers that leads to higher power consumption.

Traditionally, electric energy systems are unidirectional in operation and top-down oriented. Demand and supply balance is kept by feeding the system with large power plants (sized to hold the peak request but only for short times). This balance is crucial and should be kept for all times, and also, is under the threat of intermittent nature of renewable energy sources (whose presence and quantity largely fluctuates in time) and increasing number of the electric vehicles (which act as either capacitors or load for the grid depending on their status) [10].Thus new challenges faced by power systems operators requires new tools and sophisticated control methodologies [10].

The idea of using the load as a control medium has been adopted by the "Utilities" for a long time as a mechanism for power curve smoothing. As a result, we have seen the birth of the concept of demandside management (DSM), which refers to the desired changes in the timing and amount of the electricity demand by applying load control systems, improved energy-efficiency cycles, on-site energy storage packages and the promotion of off-peak usage of electricity [11]. Fast acting DSM tools have only recently become affordable due to the development of accessible and cheap global communication infrastructure. In addition, embedded systems now make it relatively easy to add a certain dose of "smartness" to the loads themselves. The development in this area is driven by the fact that-despite increased efficiency of electric devices-consumption is steadily rising some percent every year due to the increase in numbers of customers (i.e. both at industrial as well as household or commercial level). While generation might not be much of a problem (it is always possible to build new production facilities), it is the grid capacity that makes many of the involved stakeholders concerned [12]. Most of the losses occur during energy conversion and transport, which are functionalities of the Grid, and additionally there is the problem of limited resources, demand growth, as well as needs for production configurations that can take into account the long term sustainability factor.

However, existing studies in optimised load-balancing have limitations in the basic assumption on the relationships between losses and workloads. Existing studies and commercial solutions mainly aims at optimal distribution of work-loads between the servers. They generally assume a linear relationship between losses and workload. Thus, they propose that the best solution is simply loading of the servers up to a certain limit and then move the remaining load to the next available one. Troublingly, however, the losses are not linear due to jet effect. High load may cause higher losses. Rather than using the simple linear model, this project will use a quadratic equation model instead.

Also, the current literature only considers load optimisation among the servers. That is an optimisation in the space dimension. The GREENDC project will optimise the systems time dimension as well. The project will take a more holistic view by considering the system as a whole i.e. servers, cooling system, backup power and electrical distribution system. Here is an example of using this approach: For a unit of workload, an IDC create heat and need to cool it down. Let us assume one unit of workload requires one unit of cooling power. If we do both at the same time we need two units of power and this creates losses square of that power i.e. 4 times. If we instead overcool the system before the workload and allow slight overheat and again overcool the system after the workload, we can distribute the load in time. That means doing the same work in the longer term. That halves the losses. In our approach we will distribute the workload in space and time by utilising thermal inertia of the system as an energy storage medium. 
Finally, the GREENDC project will develop an IDC simulator in which IDC managers can evaluate different strategies to minimise energy consumption and $\mathrm{CO} 2$ emissions in the consideration of space and time dimensions as described above.

The secondments proposed in this project will allow for the above factors to be studied through simulation techniques and algorithms, resulting in important knowledge transfers between the IDCs, consortium partners, industry and academia, and through outreach activities create more awareness about the importance of these challenges for the public to take action into pushing the involved stakeholders to reduce the ecological footprint.

The specific objectives of the GREENDC project are as follows.

- defining energy demand forecasting and control problems through a systematic review of the literature on the energy optimisation in IDCs considering carbon footprint and field studies in two real world IDCs at industrial partners to develop a deeper understanding of the key variables and parameters that affect GHG emissions, service level and cost of IDC operations;

- mathematical and simulation modelling of IDC operations and developing an efficient and scalable metaheuristic optimisation techniques to guide the search in an interactive mode with the simulation module for trade-off analysis in the form of Pareto-optimal frontier;

- prototyping a decision support tool (GREENDC DSS) for industrial applications by industrial partners and other companies operating IDCs;

- testing, validating and benchmarking of the decision support tool using case studies from Turksat (TSAT) and David Holding (DAVID) that ensure minimum 10\% improvement in energy consumption of the pilot IDCs in Turkey and Bulgaria;

- dissemination of results to practitioners as well as academic beneficiaries through conferences, workshops and academic publications meeting minimum threshold as defined in Table B2.1 and Table B2.2;

- providing a new and lasting collaboration opportunities between Brunel Universitly London (UBRUN), TSAT, Gebze Technical University (GTU), DAVID and LK Knowledge Engineering (LKKE) through 215 PMs of cross-sectorial knowledge exchange by 16 experienced and 13 early-stage researchers; and

- training early stage researchers through their participation in the research and technological development activities of the project.

\section{LITERTURE REVIEW}

In the literature, Demand Side Management (DSM) tools can be categorized in the following three-fold fashion. The first one has long been realized through Time of Use (ToU) price mechanism by adjusting the tariffs. ToU pricing can simply be defined as "varying the price depending on the time-of-day" and be implemented by various utilities all around the World [15].

The second DSM mechanism is utilized through market mechanism[5],[16],[17]. The electricity price is determined by the bidders one day in advance and they are penalized or rewarded depending on their bidding accuracy. Thus accurate estimation of prospected energy demand is an important winning factor for the bidders. However, the IT service energy consumption can be delayed, migrated or completely shaded, through flexible contracts, when the market price converges to a higher equilibrium point. This requires precise estimation algorithms for the computation work load and its related energy requirements. In addition, the operational cost can be minimised by using various optimisation algorithms as proven by various researcher. Market clearing procedure (MCP) is determined using by Lagrangian relaxation and mixed-integer linear programming in this study [16]. Workload shifting is one of the techniques used for reducing the total energy consumption and related cost. In this technique, computing tasks are postponed to the future by the servers in the IDCs. The system is kept temporary idle or completely shut down [18],[19],[20]. In reference [18] stochastic optimization based algorithms were developed and used based on 26 years of data from Fort Collins Utilities in Colorado obtained by running the Coincident Peak Pricing(CPP) program. Reference [19] defines schematically how the load shifting technique is used. The author improved online algorithm for load shifting. This algorithm decides whether the workload should be waited in the queue or be directed to a server in the DC. In reference [20] CPU resource technique was used to power management of state of active servers in the DC. Monte Carlo set up is used as simulation tool. This work does not care about immediacy of serviced workload since delay in the process is not an issue for this particular case. The second technique to be used is the load migration which refers to geographically shifting of computing load to another DC with lower energy prices from a DC with 
higher prices [18],[19],[20],[21]. Load migration technique was used in [21] to show that it is beneficial both utilities and DC operations. The load shedding method is based on temporary sleeping or shutting down of the servers that are running at idle in order to reduce the power consumption. Generally speaking, state of sleeping or shedding server consume energy lower than state of idle [21]. However, the servers consume maximum power in the wake-up time to serve again. Therefore, various methods have been developed in which case the decision would be advantageous to "sleeping" state or "idle" state [14],[20],[21].

The third option for DSM is formulated by demand side frequency control actions. This is realised through sending a price signal by means of frequency changes. Power system frequency is almost constant and maintained through large power plants. The controller time constants of the large power plants vary between a few seconds up to a few tens of minutes [23]. Thus if successful communication infrastructure and control devices are available, system frequency/demand can be controlled by means of demand adjustment. This simply manifests itself as "increase the load when the system frequency tends to be higher and decrease the load vice versa" [24]. In this case the IT loads have promising features in implementing such actions. Particularly, cooling system can be adjusted to work with a larger band [25]. As a matter of fact, the system is overcooled when the system frequency is higher and slight overheating is allowed when system frequency become lower. IT system workload can also be shifted in time, or migrated to the other location where system frequency is in suitable range. The back-up systems in IDCs can also provide an opportunity to curtail the energy consumptions when the system price is high. IDCs with large and flexible loads are good candidates for demand side frequency control actions. IDCs have to contribute with active participation in DSM. However, this participation is much more challenging and also studies on this aspect are ongoing [26].

The existing literature reports the use of linear models for the estimation of energy requirements of the IT devices. Generally speaking, most researchers [4],[5],[13],[27] assume that each DC use homogenous servers and configurations, resulting in power consumption profile which are assumed to be constant for each server at every location often also with the same power consumption model. In [13], energy consumption $(P)$ was calculated by two variables: CPU utilization and frequency. According to this, power consumption has a linear relationship with these two variables.

In refer [13], a linear programming formulation to minimize the electricity cost was developed by using leverage Brenner's fast polynomial-time algorithm. Also IT workload modelling is based on predicting workload in the future. Thus, generally the stochastic process modelling is used to estimate uncertain workload in the future such as Markov Modulated Poisson Process (MMPP) [28] and Markovian Arrival Processes (MAP) [29]. Generally, [5],[16] developed mixed integer programming (MIP)-based model to demand management of IDCs. A MIP based model minimizes electricity cost and optimizes IDC server scheduling. Also Quality of Service requirements of batch computing and reliability of the IDC system are major concern. There is another similar work in [2], focusing on minimizing power consumption in IDCs, which has developed a new approximation called StochAstic power reduction schEme (SAVE) based on Lyapunov optimization framework [30]. Save has got three algorithms: front end routing, back end server management and queue update. Also for each algorithm equations different theorems were used such as Markovian, Caratheodory theorem. The other method is Lagrange multipliers used for active energy optimization [27].

It should be appreciated that the energy requirements of IT devices (and their related cooling needs) are heavily influenced by ambient temperature and building structures. It should also be emphasised that relationship between IT workload and energy requirement is non-linear, something that is being omitted by the existing literature. Thus accurate estimation of DCs energy demand requires non-linear models and reliable optimization techniques. Given the fact that accurate energy consumption estimation is crucial for the optimal operation of the system, this project will use heuristic or meta heuristic optimization techniques for system optimization [31]. This approach will also significantly benefit from the knowledge transfer activities between industrial and academic partners to ensure that models are based on actual data. The intention is to use artificial bee colony algorithm (ABC), which is a swarm based optimization methods, which the partners have significant experience is using (example is GTU using it for power systems optimization in other projects such as Distributed Generation allocation in electrical distribution systems).

The GREENDC project represents an opportunity to develop better understanding of load side frequency control. Various electrical distribution system operators have shown interest for this concept, and it is thus expected that in the near future customers will be offered special contracts that imply the possibility to participate to demand side frequency control schemes. It can intuitively be said 
that data centres can actively be part of such systems by migrating and delaying IT workload. Expanding the cooling systems operation-band will provide huge opportunities for energy storage. Emergency back-up systems also have promising features and active usage of such systems without causing any reliability issue should further be exploited. Integration of renewable power generation [20] will be another opportunity since cooling systems, immigration and delaying of IT work load plus conventional backup systems may be used as storage mediums to overcome intermittency of renewables. Study, simulation and knowledge transfer of all these aspects will be carried out in this project.

The main contributions of the GREENDC project will be better understanding, analysis and simulations of:

- Non-linear modelling of IT energy requirements

- Usage of Heuristic/meat heuristic optimization techniques to solve the non-linear optimization problem

- Exploiting of DCs energy storage capability to overcome intermittency issues of renewables via an IDC simulator.

All information and results will be organized, commented and structured to provide valuable insight on the related cost/benefit implications so as to help DC managers to acquire the knowledge to take better, more informed and responsible decisions.

\section{METHODOLOGY}

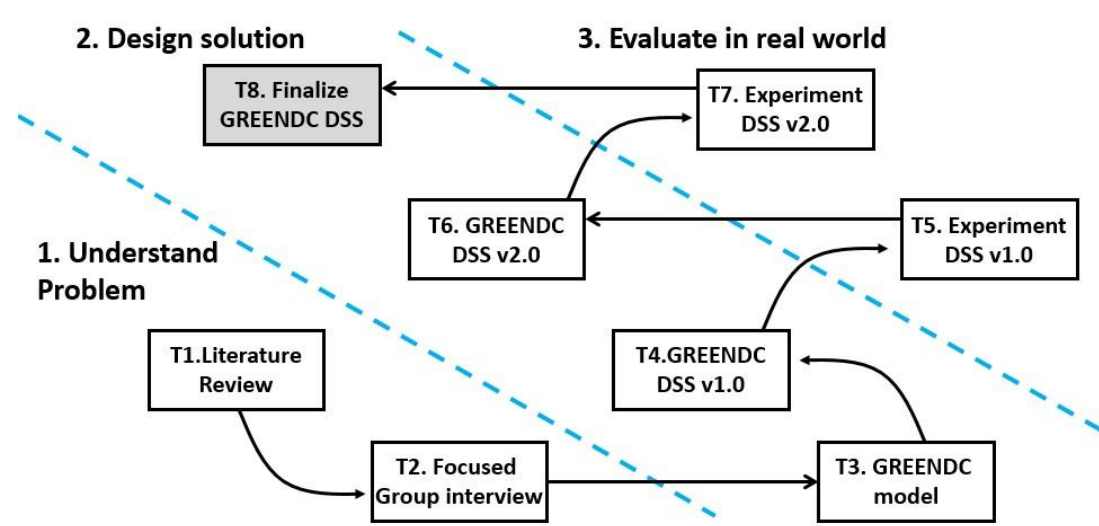

Figure 1 Design science approach in GREENDC project
The research will take a design science approach to deliver a managerial solution. Design science comprises the aim of designing, implementing, and testing a novel solution for managerial problems. The design science method is defined as including the following activities: identification and clear description of a relevant organisational problem; demonstration that no adequate solution exists; development and

presentation of novel artefacts (models, constructs, methods, or instantiations) that address the problem; rigorous evaluation of the artefacts enabling the assessment of its utility; articulation of the value added to the domain knowledge base and to practice; and explanation of the implications for management and practice. A novel artefact that addresses the target managerial problems is also known as a design proposition, which corresponds to a causal research model in explanatory science approach [32]. A design proposition links a specific intervention to a specific organisational outcome. In the context of this study, the proposed decision support system for finding strategies to minimize $\mathrm{CO} 2$ emissions and total energy consumption by DCs is considered as a design proposition (intervention) to improve organisational performance (green service level improvement and cost reduction). Due to the inter-disciplinary nature, collaboration between academic and industrial partners is inevitable and design science approach provides a systematic method to achieve the research goal. Figure B1.4 shows how the design science approach is applied in GREENDC project. There are three major interrelated phases: understanding the problem; designing the solution; and evaluating the design in a real world setting. Two tasks will be implemented for understanding the target problem: literature review and focused group interviews. For these tasks, UBRUN and GTU will review related mathematical models and algorithms mostly used in DC management in Task 1 (T1). The literature on the optimisation of energy forecasting and simulation considering carbon footprint will be reviewed and supplemented with field studies at the industrial partners (TSAT and DAVID). The outcome of T1 will 
be a report that summarises the findings in the literature. In Task 2 (T2), all partners will conduct focused group interviews in TSAT to confirm the findings of the literature review report with the practitioners in TSAT's DC. This will allow academic partners (UBRUN and GTU) to develop a deep understanding of the key variables, parameters and decision criteria's involved in managing DCs.

In the design solution phase, a mathematical model of energy forecasting and distribution using the DCs of the industrial partners to schedule energy distribution incorporating stochastic parameters will be developed to minimise the carbon footprint and optimise service level and cost as key decision objectives. In Task 3 (T3), a multi-objective metaheuristic will be developed to guide the search in an interactive mode with the simulation module to find Pareto optimal frontier of the optimisation problem. TSAT will provide real business process data whilst academic partners will develop the optimisation algorithms and simulation. At the same time, industrial partners (LKKE and DAVID) will provide software engineering knowledge for developing a DSS and leads Task 4 (developing GREENDC DSS version 1.0). All partners will collaborate for the design of GREENDC DSS in T4 so that the outcome of T3 can be easily integrated within the DSS later. The output of T3 and T4 will be the first version of GREENDC DSS which has basic user interface for getting user input and presenting the output. LKKE will provide a component based architecture of GREENDC; DAVID web based user interface; UBRUN IDC simulator; and GTU energy demand forecasting and load balancing module.

Once T4 produces GREENDC DSS version 1.0, the third phase (evaluating the design in the real world) will commence with the activation of Task 5 (experiment of the DSS version 1.0). From this time onwards, the two phases will interact in sequence to update the design (in the design phase) according to the feedbacks from the experiments (in the evaluation phase). The evaluation phase aims to test the developed metaheuristic optimisation technique by applying it to real operations for energy demands and distribution in TSAT. To this end, the algorithm developed in Task 3 will be applied to real world problem as defined by TSAT. The first draft of GREENDC DSS will be passed to TSAT and DAVID along with a training program which will allow TSAT and DAVID staff to use the system with minimum support from other partners. The system will be used by TSAT and DAVID for three months during the field trial (T5). After the trial, UBRUN and GTU will conduct focused group interviews to derive qualitative and quantitative evaluation of the first version of GREENDC DSS. Qualitative evaluation will focus on perceived usefulness and ease of use of the system for supporting DC management in TSAT. The quantitative evaluation will focus on achieving the goal of the DSS: reduced carbon-footprint and total cost along with improved service level. The completion of the experiments and focused group interviews will lead to revising the algorithms and GREENDC DSS (T6) which will result in GREENDC DSS version 2.0. The new version will be enhanced from the user interface as well as algorithmic performance perspectives. It will support all the functionalities required for monitoring the emission of carbon-footprint, service improvement and cost reduction in different simulation scenarios considering the current operation contexts of TSAT. Once the DSS 2.0 has been tested for a longer period of time in T7, the GREENDC DSS will be finalised in the final task (T8).

The activities of the GREENDC project are grouped into five work packages (WPs). WP1 aims at defining requirements for IDC managers and provide feedbacks on the GREENDC DSS. Partners will collaborate to develop energy demand forecasting and simulation model based on the feedback from TSAT and DAVID. WP2 is concerned with developing the GREENDC and develop managerial strategies for energy efficient IDC operation. WP3 contains tasks with regard to training secondment staff at the host partner in relation to the project objectives. Tasks in WP4 are concerned with dissemination of the GREENDC project and its outcomes to a wide range of audiences. Finally, WP5 contains project management tasks.

\section{EXPECTED IMPACTS AND CONCLUSION}

This paper proposed the GREENDC approach to reducing CO2 emissions and energy consumption from data centres based on non-linear modelling and simulation approach. In particular, this paper proposed an innovative knowledge exchange approach between industries and academia to achieve the project objectives.

The GREENDC project is expected to provide individual researchers of partner institutes with opportunity to enhance their research and innovation skills. 
UBRUN will engage three experienced researchers and four early stage researchers, who are working on their PhD degrees. The secondments of three experienced researchers at DAVID and TSAT, which are operating IDCs, will provide them with opportunity to apply their knowledge to simulations and sustainable business operations in relation to industrial problems. A successful achievement of project objectives also allows them to develop an impact case to be submitted to REF 2020 which is a research capacity evaluation for UK universities. On the other hand, the secondments of the early stage researchers of UBRUN will allow them to gain working experiences from the industry as well as collecting data for their PhD studies. In particular, the four PhD students are all working on green practices in business operations for their PhDs. Thus, taking part in the GREENDC project will help improve their understanding about how sustainability issues affect the acceptance of Green IS and operation of IDCs and managers. S Taskafa and S Lekhavat are working on simulation methods and will use the outcomes of their work in DAVID and TSAT as a part of their $\mathrm{PhD}$ dissertation.

DAVID will send three experienced researchers and three early stage researchers to UBRUN (UK) and GTU (Turkey). The experienced researchers have managed EU projects such as OASIS previously. The GREENDC project will allow them to further enhance their skills and expertise in a new context and extend their network of partners. More specifically, the GREENDC project is expected to extend their knowledge about Green ICT tool development and project management. In regards to the three early stage researchers, the project will allow them to work on IDC simulation and load-balancing algorithms and in the process gain new knowledge and insights from the experienced researchers belonging to the other partners. Although their involvement in the project is mainly focused on front-end user interfaces, the integration task together with the academic partners will allow them to gain critical knowledge regarding the logic of simulator and optimization algorithms.

GTU will involve four academic staff members and four PhD students through the secondments. The secondments of academic staff at DAVID and LKKE will enable them to extend their competences and research profile to an international context. The secondments at the industrial partners are expected to provide them with a more practical understanding of real world problems, while at the same time directly apply, verify and develop their academic knowledge and skills. The $\mathrm{PhD}$ students will have a unique opportunity to work in industrial companies across Europe and apply not only engineering techniques to real world problems, but also gain vital experience of the DCs business operations. Without a doubt, the experience of working in European companies will strengthen their CVs and future career prospects.

TSAT will send six experienced researchers to UBRUN. The staff members are all currently working on improving energy efficiency of IDCs for the provision of eGovernment services. In this context, the secondment at UBRUN will provide them with a more robust scientific approach for data collection and applying data analytics techniques to measure and improve IDC energy efficiency. Also, attending diverse seminars and workshops provided by the Engineering and Business schools at UBRUN will allow them learn new techniques and scientific approaches for software development and maintenance of IDCs operations.

LKKE will send two experienced researchers to GTU. Their work in GTU will allow them to gain knowledge and expertise about electrical engineering processes in relation to how the humidity, temperature, and load-balance affect the electricity consumption from computing servers. Moreover, they will be able to work in a different economic, cultural and social environment (Turkey), and in so doing gaining insights of opportunities and challenges in a new market, which, in turn, can translate to new business opportunities, as well as long-term collaboration between the involved institutions in the project.

\section{ACKNOWLEDGEMENTS}

The GREENDC project has received funding from the European Union's Horizon 2020 research and innovation programme under grant agreement No 734273.

\section{REFERENCES}

[1] G. Jung, M. a. Hiltunen, K. R. Joshi, R. D. Schlichting, and C. Pu, "Mistral: Dynamically managing power, performance, and adaptation cost in cloud infrastructures," Proc. - Int. Conf. Distrib. Comput. Syst., pp. 62-73, 2010. 
[2] Y. Yuan, H. Longbo, A. Sharma, L. Golubchik, M. Neely, Y. Yao, L. Huang, A. Sharma, L. Golubchik, and M. Neely, "Data centers power reduction: A two time scale approach for delay tolerant workloads," in INFOCOM, 2012 Proceedings IEEE, 2012, pp. 1431-1439.

[3] R. MILLER, "Who Has the Most Web Servers?," 2009. [Online]. Available: http://www.datacenterknowledge.com/archives/2009/0.

[4] L. Rao, X. Liu, L. Xie, W. Liu, R. Lei, L. Xue, X. Le, and L. Wenyu, "Minimizing Electricity Cost: Optimization of Distributed Internet Data Centers in a Multi-Electricity-Market Environment," in INFOCOM, 2010 Proceedings IEEE, 2010, pp. 1-9.

[5] J. Li, Z. Bao, Z. Li, S. Member, L. Jie, B. Zhen, and L. Zuyi, "Modeling Demand Response Capability by Internet Data Centers Processing Batch Computing Jobs," Smart Grid, IEEE Trans., vol. 6, no. 2, pp. 737-747, 2015.

[6] R. Raghavendra, P. Ranganathan, V. Talwar, Z. K. Wang, and X. Y. Zhu, "No 'power' struggles: Coordinated multi-level power management for the data center," Acm Sigplan Not., vol. 43, no. 3, pp. 48-59, 2008.

[7] U.S. Environmental Protection Agency and U. S. E. P. Agency, "Report to congress on server and data center energy efficiency: Public law 109-431," EPA Energy Star Progr., p. 133, 2007.

[8] A. Qureshi, R. Weber, H. Balakrishnan, J. GTUtag, and B. Maggs, "Cutting the Electric Bill for Internet-Scale Systems," Sigcomm 2009, pp. 123-134, 2009.

[9] P. Bertoldi, "European Policies for Energy Efficiency in ICT," JRC Eur. Comm.

[10] P. Palensky and D. Dietrich, "Demand Side Management: Demand Response, Intelligent Energy Systems, and Smart Loads," Ind. Informatics, IEEE Trans., vol. 7, no. 3, pp. 381-388, 2011.

[11] M. A. Al-Iriani, "Climate-related electricity demand-side management in oil-exporting countries the case of the United Arab Emirates," Energy Policy, vol. 33, no. 18, pp. 2350-2360, 2005.

[12] M. Prodanovic and T. C. Green, "High-Quality Power Generation Through Distributed Control of a Power Park Microgrid," Ind. Electron. IEEE Trans., vol. 53, no. 5, pp. 1471-1482, 2006.

[13] J. Yao, X. Liu, W. He, and A. Rahman, "Dynamic control of electricity cost with power demand smoothing and peak shaving for distributed internet data centers," Proc. - Int. Conf. Distrib. Comput. Syst., pp. 416-424, 2012.

[14] R. Stanojevic and R. Shorten, "Distributed Dynamic Speed Scaling," in INFOCOM, 2010 Proceedings IEEE, 2010, pp. 1-5.

[15] R. Verzijlbergh, L. J. De Vries, and Z. Lukszo, "Renewable Energy Sources and Responsive Demand. Do We Need Congestion Management in the Distribution Grid?," IEEE Trans. Power Syst., vol. 29, no. 5, pp. 2119-2128, 2014.

[16] J. M. Arroyo and A. J. Conejo, "Multiperiod auction for a pool-based electricity market," IEEE Trans. Power Syst., vol. 17, no. 4, pp. 1225-1231, 2002.

[17] K. C. Lee, H. Lee, N. Lee, and J. Lim, "An agent-based fuzzy cognitive map approach to the strategic marketing planning for industrial firms," Ind. Mark. Manag., vol. 42, no. 4, pp. 552-563, 2013.

[18] Z. Liu, A. Wierman, Y. Chen, B. Razon, and N. Chen, "Data center demand response: Avoiding the coincident peak via workload shifting and local generation," Perform. Eval., vol. 70, no. 10, pp. 770-791, 2013.

[19] E. Kayaaslan, B. B. Cambazoglu, R. Blanco, F. P. Junqueira, C. Aykanat, and B. B. C. E. Kayaaslan R. Blanco, F. P. Junqueira, and C. Aykanat, "Energy-price-driven query processing in multi-center web search engines," Proc. 34th Int. ACM SIGIR Conf. Res. Dev. Inf. - SIGIR '11, pp. 983-992, 2011. 
[20] H. Chen and M. C. Caramanis, "Reducing the Data Center Electricity Costs Through Participation in Smart Grid Programs."

[21] D. Chiu, C. Stewart, B. McManus, and C. S. D. Chiu and B. McManus, "Electric grid balancing through lowcost workload migration," ACM SIGMETRICS Perform. Eval. Rev., vol. 40, no. 3, pp. 48-52, 2012.

[22] C. Isci, S. McIntosh, J. Kephart, R. Das, and S. M. C. Isci J. Kephart, "Agile, efficient virtualization power management with low-latency server power states," Proc. 40th ..., pp. 96$107,2013$.

[23] F. D. Galiana, F. Bouffard, J. M. Arroyo, and J. F. Restrepo, "Scheduling and Pricing of Coupled Energy and Primary, Secondary, and Tertiary Reserves," Proc. IEEE, vol. 93, no. 11, pp. 19701983, 2005.

[24] F. Shariatzadeh, P. Mandal, and A. K. Srivastava, "Demand response for sustainable energy systems: A review, application and implementation strategy," Renew. Sustain. Energy Rev., vol. 45, pp. 343-350, 2015.

[25] C. Bash and G. Forman, "Cool job allocation: Measuring the power savings of placing jobs at cooling-efficient locations in the data center," Usenix Assoc. Proc. 2007 Usenix Annu. Tech. Conf., pp. 363-368, 2007.

[26] B. Aksanli and T. Rosing, "Providing regulation services and managing data center peak power budgets," pp. 1-4, 2014.

[27] T. Horvath and K. Skadron, "Multi-mode energy management for multi-tier server clusters," Proc. 17th Int. Conf. Parallel Archit. Compil. Tech., pp. 270-279, 2008.

[28] G. Latouche and V. Ramaswami, "Introduction to Matrix Analytic Methods in Stochastic Modeling," Technometrics, vol. 43, no. 3, pp. 379-380, 1999.

[29] S. Pacheco-Sanchez, G. Casale, B. Scotney, S. McClean, G. Parr, and S. Dawson, "Markovian workload characterization for QoS prediction in the cloud," Proc. - 2011 IEEE 4th Int. Conf. Cloud Comput. CLOUD 2011, pp. 147-154, 2011.

[30] M. A. Leal and J. S. Gibson, "A first-order Lyapunov robustness method for linear systems with uncertain parameters," IEEE Trans. Automat. Contr., vol. 35, no. 9, 1990.

[31] A. Antoniou and W. S. Lu, Practical optimization: Algorithms and engineering applications. 2007.

[32] Romme, A.G.L. (2003). Making a Difference: Organization as Design. Organization Science 14, $558-573$ 